\title{
Method of Rapid Assessment of Photocatalytic Activities of Self-Cleaning Films
}

\author{
Andrew Mills,* Jishun Wang, and Mark McGrady \\ Department of Pure \& Applied Chemistry, University of Strathclyde, 295 Cathedral Street, \\ Glasgow G1 1XL, U.K.
}

Received: June 8, 2006; In Final Form: July 17, 2006

\begin{abstract}
An ink, comprising the redox dye resazurin $(\mathrm{Rz})$ and the sacrificial electron donor glycerol, is shown to be capable of the rapid assessment of the photocatalytic activities of self-cleaning films. In the key initial stage of photocatalysis the ink changes from blue to pink. Prolonged irradiation bleaches the ink and eventually mineralizes it. The kinetics of the initial photoinduced color change is studied as a function of UV irradiance, [glycerol], [Rz], and temperature. The results reveal an apparent approximate quantum yield of $3.5 \times 10^{-3}$ and an initial rate, $r_{\mathrm{i}}$, which increases with [glycerol] and decreases with [Rz]. It is proposed that the reduction of $\mathrm{Rz}$, dispersed throughout the thick (ca. $590 \mathrm{~nm}$ ) indicator film, may take place either via the diffusion of the dye molecules in the ink film to the surface of the underlying semiconductor layer and their subsequent reaction with photogenerated electrons and/or via the diffusion of $\alpha$-hydroxyalkyl radicals, produced by the oxidation of the glycerol by photogenerated holes, or hydroxy radicals, away from the surface of the semiconductor into the ink film and their subsequent reaction with the dye molecules therein. The decrease in $r_{\mathrm{i}}$ with [Rz] appears to be due to dimer formation, with the latter impeding the reduction process. The activation energy for the initial color-change process is low, ca. $9.1 \pm 0.1 \mathrm{~kJ} \mathrm{~mol}^{-1}$ and not unlike many other photocatalytic processes. The initial rate of dye reduction appears to be directly related to the rate of destruction of stearic acid. The ink can be applied by spin-coating, stamping, or writing, using a felt-tip pen. The efficacy of such an ink for assessing the photocatalytic activity of any photocatalytic film, including those employed on commercial self-cleaning glasses, tiles, and paving stones, is discussed briefly.
\end{abstract}

\section{Introduction}

The basic process of semiconductor photocatalysis, SPC, can be summarized as follows:

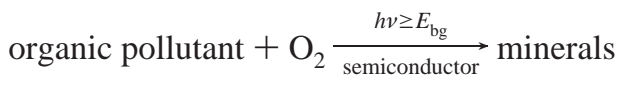

Here $E_{\mathrm{bg}}$ is the band gap energy of the semiconductor. ${ }^{1}$ Invariably the semiconductor is anatase titanium dioxide, because of its chemical and biological inertness, mechanical toughness, high photocatalytic activity, and low cost. ${ }^{1}$ Extensive research into SPC using $\mathrm{TiO}_{2}$ has shown that it is able to photocatalyze what is effectively the cold combustion of a wide range of organic materials, including many pesticides, surfactants, and carcinogens. ${ }^{1}$ As a consequence, many commercial products have emerged in recent years, including water and air purification systems and self-cleaning tiles and glasses. ${ }^{2}$ The latter in particular have met with significant commercial success and are now sold worldwide by most of the major glass manufacturers including the following (with the trade name of their commercial product in parentheses): Pilkington Glass (Activ); St-Gobain (Bioclean); PPG (SunClean); AFG (Radiance Ti). ${ }^{3,4}$ In most examples of SPC commercial products, the photocatalyst employed is a film of anatase titania; in selfcleaning glass this is usually deposited on the glass as a thin film (typically $15-20 \mathrm{~nm}$ ) by a CVD technique. ${ }^{4}$

Photocatalyst films on glass are self-cleaning in that most of the organic pollutants that go to make up the dirt and grime

* To whom correspondence should be addressed. E-mail: a.mills@ strath.ac.uk. Fax: + 44141548 4822. Tel: + 441415482458. that deposit on window glass are readily mineralized by oxygen via the photocatalytic process (eq 1). These films are also more wettable by water, i.e., hydrophilic, after UV irradiation, making it difficult for hydrophobic organic pollutants to stick on the film's surface. ${ }^{5,6}$ Even if some pollutants are able to adhere, albeit tentatively, on such a surface, they are then readily removed by rinsing with (rain) water. ${ }^{7}$ Both SPC and UVinduced hydrophilicity contribute to the overall self-cleaning functionality of the titania coating, and although there is some controversy as to how UV-induced hydrophilicity and SPC activity are related, it appears to be generally true that any titania film which is SPC active also exhibits UV-induced hydrophilicity. Thus, for a fully functioning, self-cleaning glass, it is essential that the coating exhibits SPC activity. Surprisingly, to date, the assessment of this activity remains generally nontrivial and slow.

For example, a popular method for assessing the SPC activity of a semiconductor photocatalyst film, such as a piece of selfcleaning glass, is the stearic acid test, ${ }^{8-12}$ in which a thin layer of stearic acid is deposited onto the film and its photocatalytic destruction monitored under known illumination conditions:

$$
\mathrm{CH}_{3}\left(\mathrm{CH}_{2}\right)_{16} \mathrm{CO}_{2} \mathrm{H}+26 \mathrm{O}_{2} \underset{\text { semiconductor }}{\stackrel{h v \geq E_{\mathrm{bg}}}{\longrightarrow}} 18 \mathrm{CO}_{2}+18 \mathrm{H}_{2} \mathrm{O}
$$

The destruction of stearic acid can be monitored via the amount of $\mathrm{CO}_{2}$ or $\mathrm{H}_{2} \mathrm{O}$ generated using gas chromatography ${ }^{9}$ or through the change in thickness of the stearic acid film using ellipsometry. ${ }^{12}$ However the generally preferred ${ }^{4,8,10,11}$ and simplest method is by monitoring the disappearance of the infrared absorption of the stearic acid film, in the region 2500-3500 
$\mathrm{cm}^{-1}$, as a function of time. The stearic acid test is attractive because the stearic acid is like many of the waxy, hydrophobic solids that adhere to glass in the real world, and the kinetics of its removal are zero-order, i.e., independent of how much is deposited, except for very thin films.

Whichever of the above analytical routes is taken, it is clear that the stearic acid test is not appropriate for making measurements in the field, since it requires at least one piece of sophisticated and expensive analytical equipment and, usually, a trained technician to run and maintain it. In addition, because most commercial self-cleaning glasses utilize only a very thin layer of titania, the kinetics of the photomineralization of stearic acid, or any other test organic, including dyes, is very slow and so it can take days, if not weeks, to destroy completely a stearic acid layer, depending upon its thickness, under solar UVA

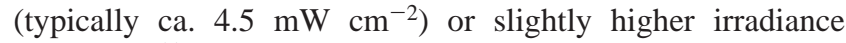
conditions. ${ }^{11}$

Clearly, the photocatalytic activity of a semiconductor film would be more accessible to assessment if it were associated with a color change, especially if the latter were rapid, i.e., within a few minutes of UV exposure. Researchers have tried staining SPC films directly with dyes, ${ }^{13,14}$ although little dye sticks to the surface, or, with more success, incorporating dyes in deposited polymer films. ${ }^{15}$ However, the major problem with these approaches is that they rely on the color change being effected by the photooxidation of the dye or dye/polymer, which is generally as slow as the oxidation of stearic acid, since both are destroyed via a common reaction intermediate, namely photogenerated hydroxyl radicals. ${ }^{15}$ Such an indicator might be adequate for assessing the SPC activities of very active, usually thick, titania films, where the kinetics of photomineralization are rapid but clearly inappropriate for most commercial, selfcleaning photocatalytic products, including glasses and tiles, that exhibit much slower rates of photocatalysis. Instead, what is required is an indicator ink that can be printed, coated, or written onto any SPC film, be it transparent or opaque, and which rapidly, i.e., within a few minutes at most, changes color upon UV activation of an underlying thin photocatalyst film. This paper reports the results of a detailed characterization study of such an ink, communicated earlier elsewhere. ${ }^{16}$

\section{Experimental Section}

Unless stated otherwise all chemicals were purchased from Aldrich Chemicals and used as received. All gases were purchased from BOC. In all the work reported here, the type of self-cleaning glass used to test the photocatalyst ink was Activ, manufactured by Pilkington Glass, which comprises a $15 \mathrm{~nm}$ thick coating of anatase titania on $4 \mathrm{~mm}$ thick window glass coated with a thin barrier layer of silicon carbide. Although Activ was used as the test sample of self-cleaning glass in this work, similar results were obtained using other commercial selfcleaning glasses, such as Bioclean and SunClean, as well as sol-gel films of a similar thickness made in house. The test pieces were usually cleaned, with a wipe of a tissue soaked in acetone of methanol, before being spun-coated with the ink, to remove any surface contaminants, such as oil or grease. However, work with the ink in a felt-tipped pen showed that such pretreatment made little difference to the rate at which the ink changed color.

The typical photocatalyst indicator ink used in this work comprised $3 \mathrm{~g}$ of a $1.5 \mathrm{wt} \%$ aqueous solution of hydroxyethyl cellulose (HEC), $0.3 \mathrm{~g}$ of glycerol, and $4 \mathrm{mg}$ of the redox dye, resazurin, Rz. ${ }^{17-19}$ Thus, the formulation of the dried ink in parts per hundred resin was as follows: 100:667:9 HEC- glycerol-resazurin, i.e., 88 wt \% glycerol in HEC. Typically, samples of glass, with and without a photocatalyst layer, were spun-coated with a few drops of this ink (500 rpm for $10 \mathrm{~s}$ ) and dried in an oven at $70{ }^{\circ} \mathrm{C}$ for $10 \mathrm{~min}$. Drying in air, rather than in an oven, did not alter the performance of the ink. A typical dried ink film was ca. $590 \mathrm{~nm}$ thick, as measured by weighing the film and using a weight-averaged density based on the amounts of HEC and glycerol present. This thickness is much greater than that of the photocatalyst layer (ca. 39 times).

All UV/vis spectra were recorded using a Thermo Electron Helios Beta UV/vis single beam spectrophotometer. Monitoring the photocatalyzed change in color of the ink as a function of temperature was achieved by placing the glass sample with ink coating on the top of a glass cell which was thermostated with circulating water and using a UV/vis spectrophotometer. The temperature of the film was measured using a digital thermometer with the probe placed on the surface of the sample. All UV irradiations were performed using a small UVA irradiation system (VWR), comprising two $4 \mathrm{~W}$ black light blue lamps (typical output: $365 \pm 20 \mathrm{~nm}$ ), with an irradiance of $7.4 \mathrm{~mW}$ $\mathrm{cm}^{-2}$ when placed directly above the sample under test.

\section{Results and Discussion}

The appearance of the ink coated onto a test glass substrate, on both plain glass and Activ, was blue, and this initial color and associated UV/visible absorption spectrum did not change upon prolonged UVA irradiation in the absence of an underlying photocatalyst film, i.e., when coated on plain glass. In contrast, as illustrated by the results in Figure 1a, the ink did change rapidly in color, i.e., within minutes, from blue to pink upon UVA irradiation when coated on a commercial sample of selfcleaning glass (Activ). Other work showed that this color change always occurred if a titania photocatalyst was present, regardless of its form (film or powder) or supporting substrate (glass, tile, paper, plastic, or metal). The results in Figure $1 \mathrm{~b}$ show that with photocatalyst film such as Activ, further irradiation (ca. $40 \mathrm{~min}$ ) of this system bleaches the ink, although, upon examination using FT-IR spectroscopy, the clear HEC/glycerol encapsulation medium remains largely unchanged over the time period associated with the photobleaching. As might be expected, given the well-established photocatalytic activity of titania, ${ }^{1}$ upon the subsequent prolonged irradiation (ca. 2 days) of this system, the clear and now colorless transparent polymer/ glycerol film is itself destroyed, as illustrated by the gradual disappearance of its infrared absorption spectrum as a function of irradiation time in Figure 1c. The photocatalytic properties of the underlying titania photocatalytic films used in this work were unaffected by this testing procedure.

As stated earlier, all previous indicator dye or dye/polymer film systems, used for illustrating SPC activity, work via a photooxidative mechanism, in which the dye is oxidatively bleached by photogenerated hydroxyl radicals while the photogenerated electrons react with oxygen to produce superoxide, another possible source of hydroxyl radicals. In contrast, the photocatalyst ink reported here operates via a photoreductive mechanism in which the photogenerated holes react irreversibly with the sacrificial electron donor (SED) present, i.e., glycerol, and the photogenerated electrons $\left(E^{\circ}\left(\mathrm{TiO}_{2}\left(\mathrm{e}^{-}\right)\right)=-0.52 \mathrm{~V}\right)^{6}$ and/or a mediating reducing species, $X$, reduce the indicator ink dye molecules, $\mathrm{D}_{\mathrm{Ox}}$, contained therein, to a differently colored form, $\mathrm{D}_{\text {Red }}$. The various steps associated with the initial color-changing ink are summarized by the reaction scheme illustrated in Figure 2.

The most likely identity of $\mathrm{X}$ is the $\alpha$-hydroxyl radical of glycerol, $\bullet \mathrm{ROH}$, which most likely moves through the HEC/ 
(a)

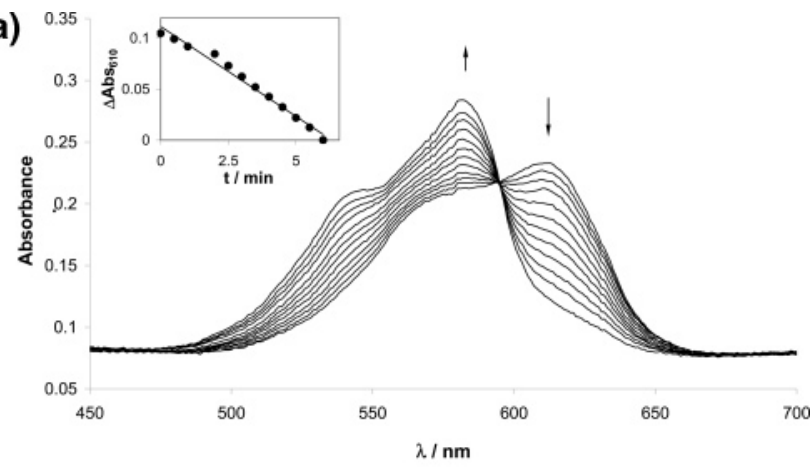

(b)

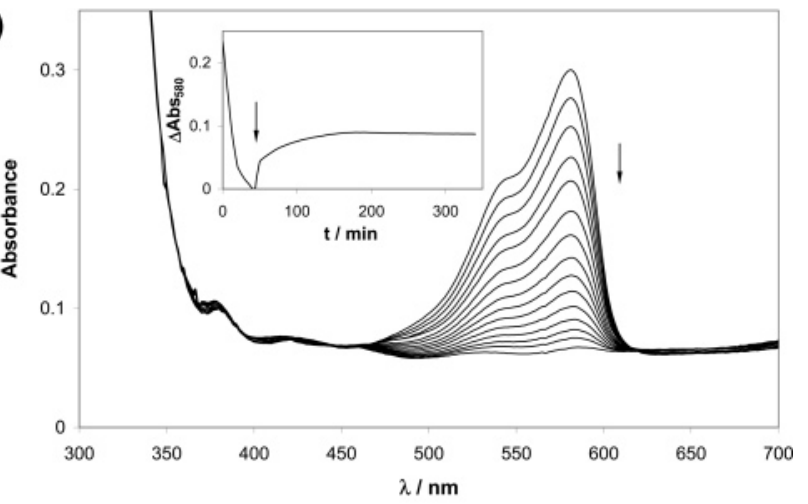

(c)

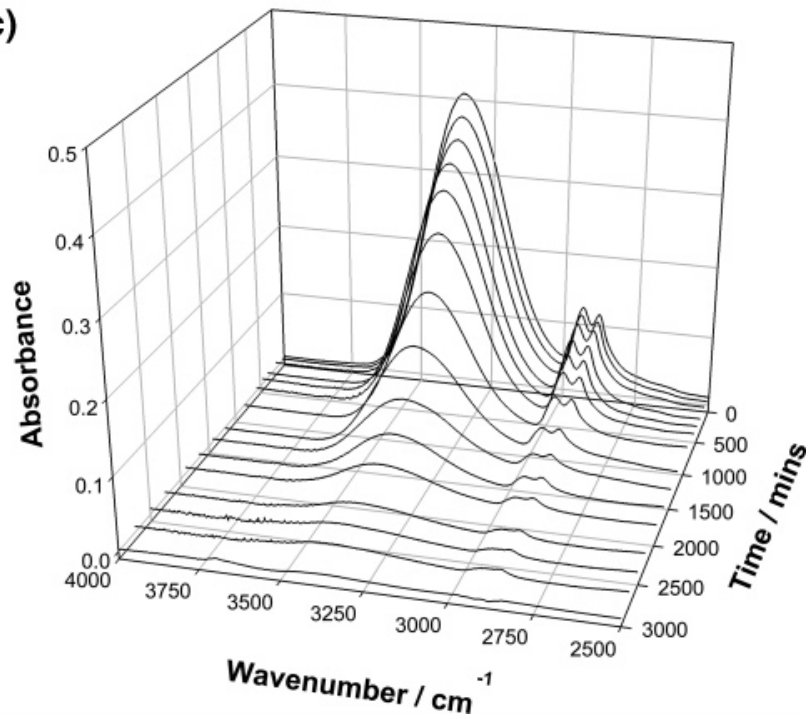

Figure 1. (a) UV/visible absorption spectra of a typical Rz photocatalyst indicator film deposited onto a sample of Activ glass recorded as a function of time of illumination with a UVA lamp, $7.4 \mathrm{~mW} \mathrm{~cm} \mathrm{~cm}^{-2}$ ( 1 spectrum $/ 30 \mathrm{~s}$ ). The arrows indicate the directions of change in the absorbance of the ink film at 610 and $580 \mathrm{~nm}$, effected by the UV irradiation, which produces the observed color change from blue to pink. The insert diagram is a plot of the change of absorbance at 610 $\mathrm{nm}, \Delta \mathrm{Abs}_{610}$, determined using the data in the main diagram, as a function of illumination time. (b) As in (a), but after the Rz dye has all been converted into $\mathrm{Rf}$ (ca. 7 min illumination) subsequent absorption changes in the film, recorded every $2 \mathrm{~min}$, as the $\mathrm{Rf}$ is converted to HRf and other colorless products. The insert diagram illustrates the observed decrease in absorbance at $580 \mathrm{~nm}$ ( $\lambda_{\max }$ for Rf) during this subsequent UV illumination (data from main diagram) and then its following recovery in the dark (start denoted by arrow). (c) Recorded FT-IR absorbance spectra of the typical Rz photocatalyst indicator film described in (a) as a function of UV irradiation time.

glycerol film via diffusion (vide infra). The generation of such a radical is probable, given that titania is known to oxidize many alcohols, including the polyalcohol ethylene glycol, by abstract-

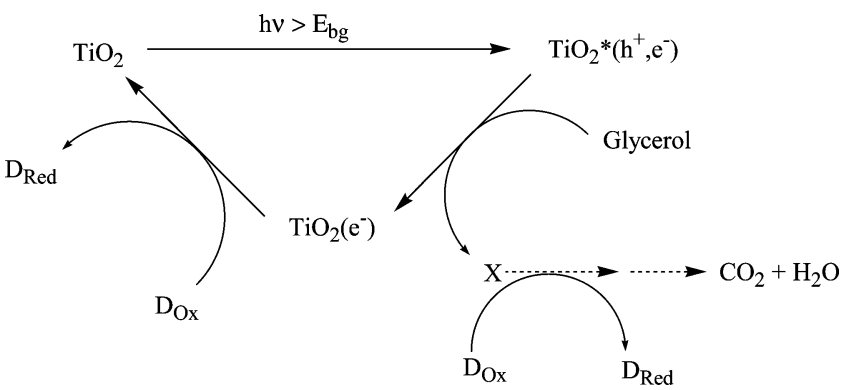

Figure 2. Reaction scheme illustrating the major processes associated with the initial reduction of the redox dye, $\mathrm{D}_{\mathrm{ox}}$, in the photocatalyst ink by a titania photocatalyst. $D_{\text {Red }}$ is the reduced form of the dye.

ing a proton on the $\alpha$-carbon atom via hydroxy radical attack or hole oxidation to generate an $\alpha$-hydroxyalkyl radical. The $\alpha$-hydroxyalkyl radical is an effective reducing agent that is able to inject an electron into the conduction band of $\mathrm{TiO}_{2}$, producing the well-known "current-doubling" effect, or reduce susceptible chemical species nearby. ${ }^{20-22}$ For example, Hilgendorff et al. ${ }^{23}$ report that the one electron, i.e., hole, oxidations of 2-propanol, ethanol, and methanol generate $\alpha$ - hydroxyalkyl radicals that are able to reduce carbon tetrachloride. In the case of glycerol, generation of the $\alpha$-hydroxyalkyl radical, $\bullet R O H$, can be summarized as

$$
\text { glycerol }+\mathrm{OH} \bullet / \mathrm{h}^{+} \rightarrow{ }^{\cdot} \mathrm{ROH}
$$

where $\bullet \mathrm{ROH}$ is an $\alpha$-hydroxyalkyl radical of glycerol.

Thus, it is proposed that the reduction of $\mathrm{Rz}$, dispersed throughout the thick (ca. $590 \mathrm{~nm}$ ) indicator film, may take place either via the diffusion of the dye molecules in the ink film to the surface of the underlying semiconductor layer and their subsequent reaction with photogenerated electrons and/or via the diffusion of $\alpha$-hydroxyalkyl radicals, produced by the oxidation of the glycerol by photogenerated holes, or hydroxy radicals, away from the surface of the semiconductor into the ink film and their subsequent reaction with the dye molecules therein.

The reduction potential of the photogenerated electrons, and apparently of $\bullet \mathrm{ROH}$, is such that many different dyes can be used in the photocatalyst ink; however, most are reversible redox dyes, such as methylene blue, MB, which react very readily with $\mathrm{O}_{2}$ in their reduced form, $\mathrm{D}_{\text {Red }}$, to regenerate the original dye and color. ${ }^{15,24-26}$ As a consequence, UV irradiation of photocatalyst indicator inks containing such dyes, on a photocatalytic film, leads to their rapid bleaching only under anaerobic conditions, a condition that is impractical with regard to the ultimate goal of being able to use the ink in the field for rapid assessment studies. In contrast, the redox dye resazurin (Rz) is a blue dye which is easily and irreversibly reduced to a pink reduced form, resorufin $(\mathrm{Rf})\left(E^{\circ}(\mathrm{Rz} / \mathrm{Rf}) \cong-0.020 \mathrm{~V}\right) .{ }^{17} \mathrm{The}$ structures of $\mathrm{Rz}$ and $\mathrm{Rf}$ are given in Figure $3 .{ }^{17} \mathrm{It}$ is this process that is responsible for the observed change in color of the initial indicator film on Activ (blue to pink) and changes in its UV/ vis spectrum, illustrated in Figure 1a upon irradiation with UV light. The reduction of $\mathrm{Rz}$ to $\mathrm{Rf}$ is irreversible, and therefore, once generated, the Rf will not be affected by the presence of oxygen. Despite the irreversibility of the dye reduction, the photogenerated electrons and $\bullet \mathrm{ROH}$ are likely to react with any dissolved oxygen present, and so it is no surprise that the rate of $\mathrm{Rz}$ photoreduction is faster (ca. 100\%) when the ink is irradiated under anaerobic compared to than aerobic conditions. However, promisingly and in marked contrast to redox dyes such as $\mathrm{MB}, \mathrm{Rz}$ is still reduced rapidly to $\mathrm{Rf}$ even under aerobic conditions, as illustrated by the spectral changes in Figure 1a. 


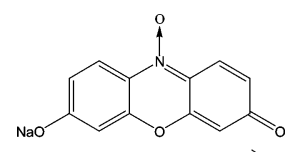

$\mathbf{R z}$

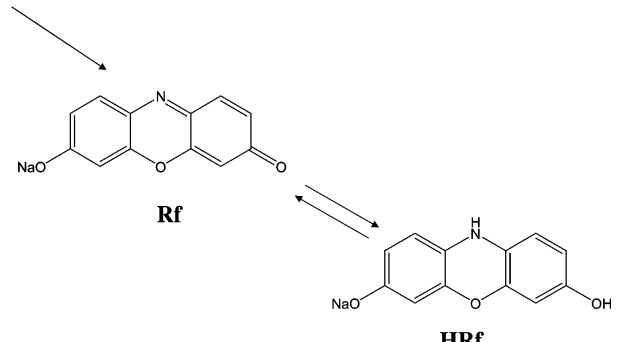

HRf

Figure 3. Structures of the indicator dye, resazurin (Rz), its initial reduced form resorufin (Rf), and further reduced form, dihydroresorufin (HRf), which can be reoxidized to Rf by ambient oxygen.

From the UV/vis spectra illustrated in Figure 1a it appears that the wavelengths of maximum absorbance, $\lambda_{\max }$, in the dried ink are $610 \mathrm{~nm}$ for $\mathrm{Rz}$ and 580 and $454 \mathrm{~nm}$ for Rf, respectively. As a consequence, in this work, the kinetics of dye color change were monitored via the change in absorbance at $610 \mathrm{~nm}$, due to Rz, i.e., $\Delta \mathrm{Abs}_{610}(t)$, as a function of irradiation time, $t$, where $\Delta \mathrm{Abs}_{610}(t)$ was taken as the difference between the absorbance of the ink film at time $t$ and after all the Rz had been converted into $\mathrm{Rf}$ (typically $\geq 7 \mathrm{~min}$ ). The insert diagram in Figure 1a illustrates the variation in $\Delta \mathrm{Abs}_{t}$ as a function of irradiation time and reveals the kinetics to be approximately zero order, over most of the reaction with respect to the resazurin concentration in the film, [Rz]. The latter finding is not too surprising given the initial high value of $[\mathrm{Rz}]$ in the dried ink film, ca. $0.06 \mathrm{~mol} \mathrm{dm}^{-3}$, and that semiconductor photocatalytic reactions often exhibit saturation type kinetics, usually welldescribed by a Langmuir-Hinshelwood type equation which predicts zero-order kinetics at high substrate concentrations.

From the UV/vis spectra illustrated in Figure 1b it appears that a Rz film on Activ, that had been photoreduced to Rf, bleaches upon further irradiation with UV light. If, as appears likely, the photobleaching process is associated with the further reduction of the dye, the most obvious product is dihydroresorufin, HRf, the structure of which is illustrated in Figure 3, but the latter is oxygen-sensitive and so the initial pink coloration due to $\mathrm{Rf}$ should recover if the photobleached film is left in the dark. ${ }^{17}$ The insert diagram in Figure $1 \mathrm{~b}$ is a plot of the change in the absorbance of the film at $580 \mathrm{~nm}$, after it has been converted to Rf, over a subsequent 40 min UV illumination period followed by a longer spell in which the irradiated film was left in the dark. This plot shows that the fully UV bleached $\mathrm{Rz}$ film, produced after $40 \mathrm{~min}$ of UV irradiation, does indeed recover some of its pink color (ca. 39\%), but not all, in the dark. Presumably the ca. $61 \%$ bleached products that are not readily reoxidized by the oxygen in air are further reduction products arising from HRf.

As noted earlier, prolonged irradiation of the system eventually leads to the complete destruction of the film, i.e., dye, polymer, and glycerol, as indicated by the FT-IR absorbance data in Figure 1c. However, the key feature of the $\mathrm{Rz}$ photocatalyst indicator ink is its ability to respond quickly (easily discernible by eye within $5 \mathrm{~min}$ ), changing blue to pink, when placed on a photocatalyst of modest activity, such as Activ, upon exposure to UVA irradiation of an intensity typical of a bright

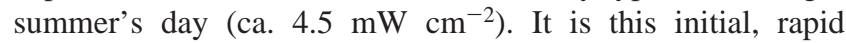
photoreduction process that is of the most interest and the focus of the following characterization process.
The variation of the initial rate of conversion of $\mathrm{Rz}$ to $\mathrm{Rf}$, i.e., $r_{\mathrm{i}}$ (units: absorbance units $\mathrm{min}^{-1}$ ), was studied as a function of incident light irradiance, $I$, over the range $0-7.4 \mathrm{~mW} \mathrm{~cm}^{-2}$, and the results are illustrated in Figure 4. From this plot the initial rate of conversion appears proportional to $I$, with a gradient of $(1.3 \pm 0.1) \times 10^{-3}$ absorbance units $\mathrm{min}^{-1} \mathrm{~mW}^{-1}$ $\mathrm{cm}^{2}$. Given that the initial value of $\Delta \mathrm{Abs}_{t}$ was typically 0.10 (see Figure 1a), equivalent to $3.5 \times 10^{-9} \mathrm{~mol}$ of $\mathrm{Rz} \mathrm{cm}^{-2}$, and that $1 \mathrm{~mW} \mathrm{~cm}^{-2}$ of $365 \mathrm{~nm}$ light is equivalent to $1.1 \times 10^{17}$ photons $\mathrm{cm}^{-2} \mathrm{~min}^{-1}$, it is possible to estimate the formal quantum efficiency of Activ for converting $\mathrm{Rz}$ into $\mathrm{Rf}$ as $(2.5$ $\pm 0.5) \times 10^{-4}$ molecules/photon. Spectral studies on Activ and CVD films suggest that the $15 \mathrm{~nm}$ layer of titania will only absorb ca. $7.2 \%$ of the incident UV light, ${ }^{11}$ indicating that the actual quantum yield for the reduction of $\mathrm{Rz}$ to $\mathrm{Rf}$ on Activ is likely to be nearer to ca. $3.5 \times 10^{-3}$. The observation that $r_{\mathrm{i}}$ is proportional to $I$ is consistent with the relatively low intensities used in this work, the low absorbance of the self-cleaning glass film, and the fact that at low absorbed light levels the reaction will be controlled by the surface reaction of the photogenerated electron-hole pairs rather than their recombination. Presumably one reason for the low quantum yield for converting $\mathrm{Rz}$ to $\mathrm{Rf}$ is because the process involves the photogeneration of reducing species, such as conductance band electrons and $\bullet \mathrm{ROH}$ which are not only able to react with the $\mathrm{Rz}$ dye molecules dispersed throughout the relatively thick HEC/glycerol film but also with any ambient oxygen present.

In another set of experiments the amount of glycerol used in formulating the indicator ink, [glycerol], was varied from 0 to $0.95 \mathrm{~g}$, i.e., from 0 to $2100 \mathrm{phr}$. The spectral properties and the variation in the initial rate of $\mathrm{Rz}$ conversion were measured for each film formulation, and the results of this work are illustrated in Figure 5 as a function of [glycerol]. This Figure 5 shows the change in the wavelength of maximum absorbance, $\lambda_{\max }$, of the film as a function of the amount of glycerol used in its formulation. From these data it appears that, in the absence or the presence of a small amount (ca. $0.05 \mathrm{~g}$ ) of glycerol, the indicator film has an absorbance maximum that is ca. $12 \mathrm{~nm}$ red-shifted compared to that of a typical indicator film (with $0.3 \mathrm{~g}$ of glycerol). Raising the glycerol content above $0.05 \mathrm{~g}$ up to glycerol levels of $\geq 0.2 \mathrm{~g}$ leads to a marked shift in $\lambda_{\max }$, and above this level of glycerol $\lambda_{\max }$ does not change with increasing glycerol level; i.e., it remains at $610 \mathrm{~nm}$. Although not quite inversely tracking the spectral changes, the variation in $r_{\mathrm{i}}$ also changes markedly with increasing glycerol level, reaching a plateau rate at ca. $\geq 0.3 \mathrm{~g}$ glycerol as illustrated by the plot of the variation in $r_{\mathrm{i}}$ as a function of the amount of glycerol added in Figure 5. These results show that in the absence of glycerol the overall process, summarized in Figure 2 , does not occur to any great extent, indicating that the polymer, HEC, is unable to act as an effective SED and that even when a small amount of glycerol is present, the latter is insufficient to promote the photocatalytic process.

The variation in the initial rate as a function of glycerol level appears related, partially at least, to the spectral changes observed for the film, which are most likely due to a modest solvatochromic effect experienced by the $\mathrm{Rz}$ dye, due to the change in the microenvironment surrounding the encapsulated dye as the level of glycerol is increased. The rates at which the dye, $\mathrm{Rz}$, and the reducing species, $\bullet \mathrm{ROH}$, are able to diffuse through the ink film to effect the reduction of the dye are also likely to be affected by any change in the microenvironment, especially given the recognized plasticizing action of glycerol in polymers such as HEC and poly(vinyl alcohol), PVA, which 


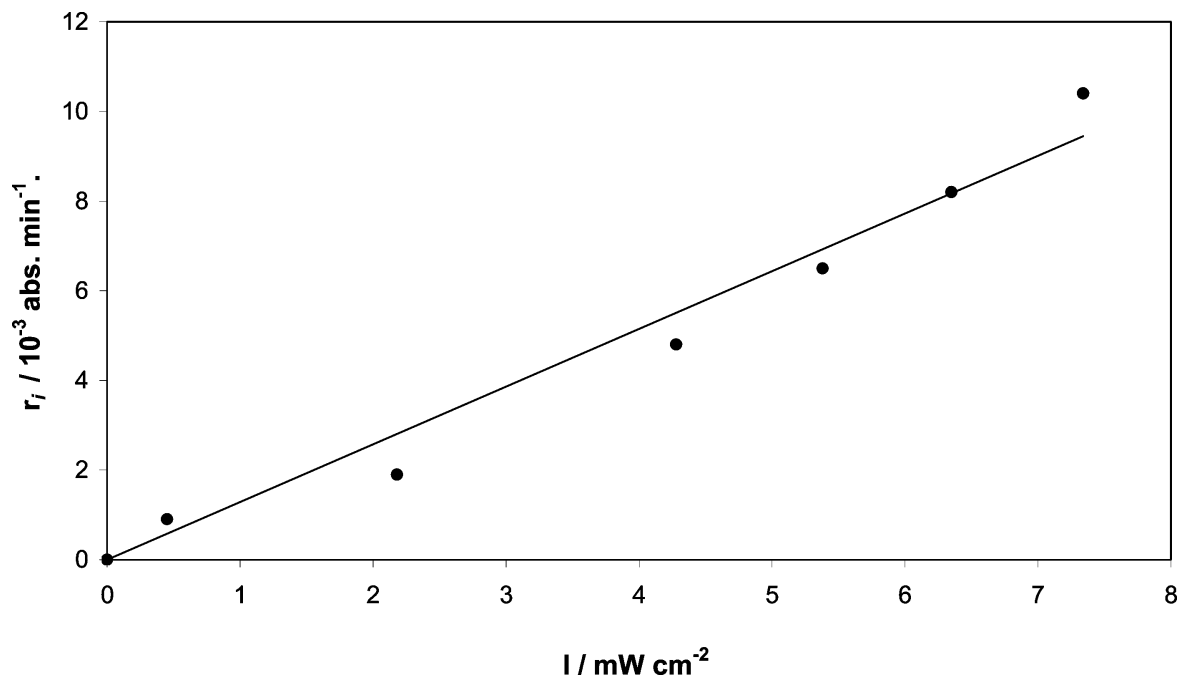

Figure 4. Plot of initial rate of Rz ink conversion to Rf, $r_{\mathrm{i}}$, as a function of incident UV light irradiance, I. The line of best fit has a gradient of $(1.3 \pm 0.1) \times 10^{-3} \mathrm{abs} \mathrm{min}^{-1}(\mathrm{~mW} \mathrm{~cm})^{-2}$.

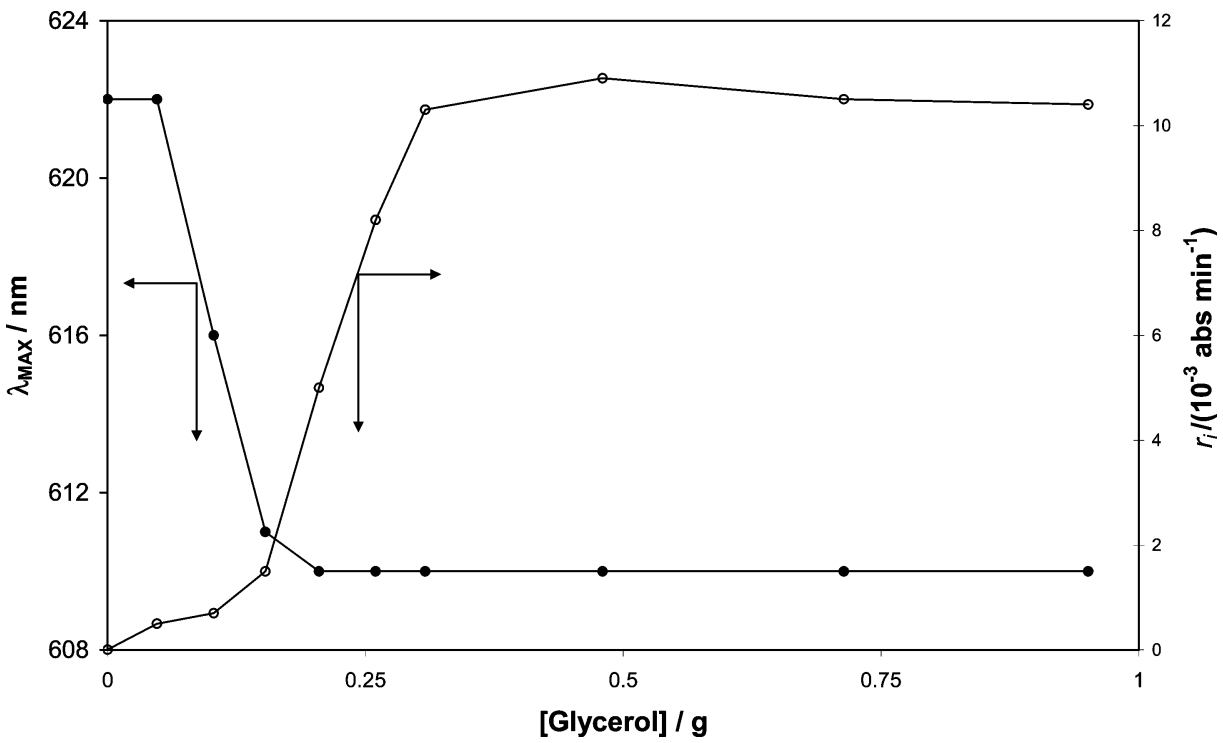

Figure 5. Plots of the variation in $\lambda_{\max }$ of the Rz ink and $r_{\mathrm{i}}$, as a function of the level of glycerol used in the formulation of the Rz photocatalyst indicator ink. All other component levels remaining fixed at their typical levels.

increases the diffusion coefficients for many chemical species encapsulated in such polymers. Indeed, Van Keuren et al. ${ }^{27}$ have reported that the value of the diffusion coefficient, $D$, for rhodamine $\mathrm{B}$, in glycerol/PVA films is increased markedly from ca. $1 \times 10^{-17}$ to $1 \times 10^{-13} \mathrm{~m}^{2} \mathrm{~s}^{-1}$ as the glycerol level is increased from 10 to $40 \mathrm{wt} \%$ and that the latter value for $D$ is similar to that expected for neat glycerol. In the dried $\mathrm{Rz}$ ink film used in this work the level of glycerol present is ca. $88 \mathrm{wt}$ $\%$, and so it appears reasonable to assume that in such films the diffusion coefficient is near to the limiting value of $1 \times$ $10^{-13} \mathrm{~m}^{2} \mathrm{~s}^{-1}$, associated with the dye in neat glycerol. It follows that the time taken for a $\mathrm{Rz}$ molecule to diffuse from the air side of the ink film to the semiconductor side will be equal to $d^{2} / 2 D=1.7 \mathrm{~s}$, given $d=590 \mathrm{~nm}$. This time is significantly less than that taken to photobleach a typical film, ca. $6 \mathrm{~min}$, see Figure 1a, and indicates that the rate-determining step for the reduction of the dye molecules is most likely not controlled solely by the rate of diffusion of either the Rz molecules to the surface of the semiconductor and/or the $\bullet \mathrm{ROH}$ radicals from the surface to the dye molecules in the ink film. However, it appears likely that the kinetics depend to some extent on the rate of diffusion of $\mathrm{Rz}$ and/or $\bullet \mathrm{ROH}$ in the glycerol/polymer film, given the marked dependence of the initial rate of dye reduction upon the level glycerol level used in the film formulation illustrated in Figure 5 and the well-established plasticizing action of glycerol on polymers, such as HEC and PVA. Presumably the leveling off of $r_{\mathrm{i}}$ at glycerol levels ca. $\geq 0.3 \mathrm{~g}$, illustrated in Figure 5, is due to the values of $D$ for $\mathrm{Rz}$ and $\bullet \mathrm{ROH}$ approaching that expected in neat glycerol. Further work is in progress to probe the mechanism of dye photoreduction. In particular, the rate of dye photoreduction is currently being studied as a function of film thickness, since this will help reveal more clearly the dependence of the kinetics upon diffusion.

The variation in $r_{\mathrm{i}}$ was also studied as a function of the concentration of $\mathrm{Rz}$ in the film, [Rz], over the range $1-8 \mathrm{mg}$ in the original formulation $\left(\equiv(1.5-12) \times 10^{-2} \mathrm{M}\right)$. The results of this work are illustrated in Figure 6 and shows that $r_{\mathrm{i}}$ decreases by a factor of ca. 4 when $[\mathrm{Rz}]$ is increased by a factor of $8 . \mathrm{Rz}$ absorbs little in the UV, and so this effect cannot be simply attributed to UV-screening by the dye; instead it appears to be associated with aggregation of the dye with increasing [Rz], where [Rz] is defined as the level of Rz used in the film formulation (units: $\mathrm{mg}$ ). This effect can be seen in the different 


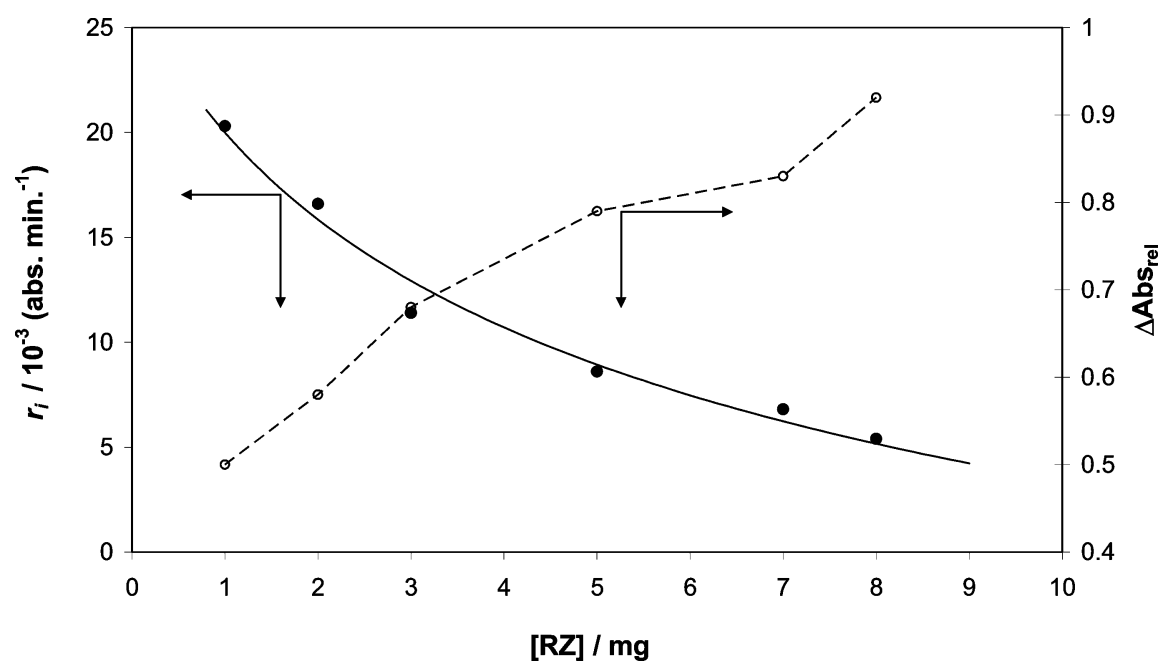

Figure 6. Plots of the $r_{\mathrm{i}}$ and the change in initial absorbance of the Rz film at $570 \mathrm{~nm}\left(\Delta \mathrm{Abs}_{\mathrm{rel}}\right)$, based on all spectra normalized so as to have a relative absorbance $=1$ at $610 \mathrm{~nm}$, as a function of the level of Rz used in the formulation of the ink, i.e., [Rz]. Units: mg; all other component levels remaining fixed at their typical levels. The solid trend line was calculated using eqs 4,5 , and 6 and $r_{\mathrm{iM}}$ and $r_{\mathrm{iD}}$ values of 27 and $-40 \times 10^{-3}$ abs units $\min ^{-1}$, respectively.

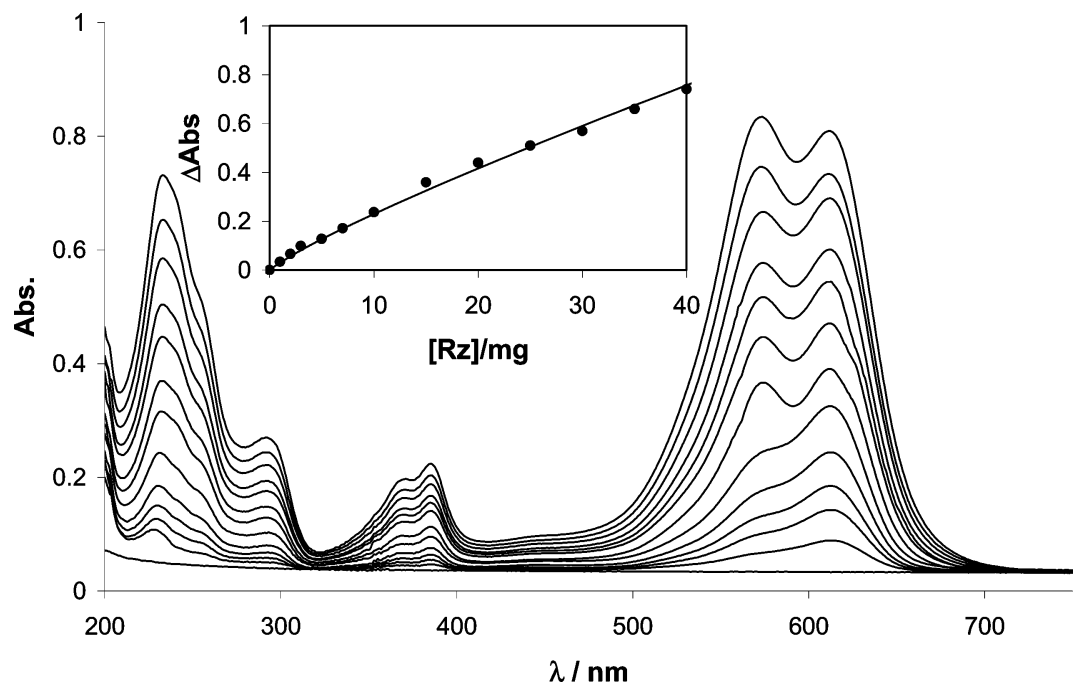

Figure 7. UV/visible absorption spectra of a series of Rz photocatalyst inks with the following different initial levels of Rz present (from bottom to top): $0,1,2,3,5,7,10,15,20,25,30,35$ and $40 \mathrm{mg}$, respectively. The levels of all the other components in the ink were fixed at those for a typical film. The insert diagram is a plot of the change in absorbance at $570 \mathrm{~nm}$ (data from main diagram) as a function of [Rz], with a trend line calculated using eq 4 , with $K=0.05 \mathrm{mg}^{-1}, \epsilon_{\mathrm{Rz}}=31300 \mathrm{M}^{-1} \mathrm{~cm}^{-1}$, and $\epsilon_{\mathrm{dimer}}=14700$ at this wavelength.

dried ink films themselves which change in color from blue (1 $\mathrm{mg} \mathrm{Rz}$ ) to purple ( $8 \mathrm{mg} \mathrm{Rz}$ ) and is completely reversible. Figure 7 illustrates the observed change in the UV/vis absorption spectra of a series of $\mathrm{Rz}$ ink films on quartz in which different amounts of $\mathrm{Rz}$ were used in their formulation. At low levels of $\mathrm{Rz}(1 \mathrm{mg})$ the shoulder peak at $570 \mathrm{~nm}$ is just visible and is similar to that seen in the visible absorbance spectrum of the monomer form of the dye in aqueous solution. At the typical level used in the film formulation ( $4 \mathrm{mg}$ of $\mathrm{Rz}$ ) this shoulder peak is much more discernible, and at $\geq 7 \mathrm{mg}$ of $\mathrm{Rz}$, it emerges as a clear peak, similar in magnitude to the main one at 610 nm.

The emergence of this shoulder peak, illustrated in Figure 6 by the variation in $\Delta \mathrm{Abs}_{\text {rel }} \mathrm{vs}[\mathrm{Rz}]$, attributed here to the formation of a dimer of $\mathrm{Rz}$, tracks very well in an inverse sense with the simultaneous decrease in $r_{\mathrm{i}}$ with increasing $\mathrm{Rz}$ level, as illustrated by the results in Figure 6 . Indeed, assuming that the spectral changes illustrated in Figure 7 are due to dimer formation and that the molar absorbtivity of the monomer of $\mathrm{Rz}$ in the film is the same as that in aqueous solution (i.e. ca. $31300 \mathrm{M}^{-1} \mathrm{~cm}^{-1}$ at its shoulder peak of $\lambda_{\max }=570 \mathrm{~nm}$, respectively), then it is possible to estimate a value for the dimerization constant, $K$, where

$$
K=[\mathrm{D}] /[\mathrm{M}]^{2}
$$

and $[\mathrm{D}]$ and $[\mathrm{M}]$ are the levels of dimer and monomer of $\mathrm{Rz}$ present in the film formulation, assuming $[\mathrm{Rz}]=[\mathrm{M}]+2[\mathrm{D}]$.

The insert diagram in Figure 7 illustrates a typical fit to the change in absorbance observed at $570 \mathrm{~nm}$ for the $\mathrm{Rz}$ films on quartz as a function of [Rz], based on eq 4 and an optimized fit to the data using a value for $K$ of $0.05 \mathrm{mg}^{-1}$, equivalent to 1.7 $\mathrm{M}^{-1}$, and values of the molar absorbtivities of the monomer and dimer of $\mathrm{Rz}$ (i.e. $\epsilon_{\mathrm{Rz}}$ and $\epsilon_{\text {Dimer }}$ ) at this wavelength of 31300 and $14700 \mathrm{M}^{-1} \mathrm{~cm}^{-1}$, respectively. Similar good fits to the spectral data using $K=0.05 \mathrm{mg}^{-1}$ were observed at all wavelengths, including $610 \mathrm{~nm}$.

In any ink film formulation, the fractions of $\mathrm{Rz}$ that are in the monomer or dimer forms, i.e., $f_{\mathrm{M}}$ and $f_{\mathrm{D}}$, respectively, are given simply by the following ratios:

$$
f_{\mathrm{M}}=[\mathrm{M}] /[\mathrm{Rz}] \quad f_{\mathrm{D}}=[\mathrm{D}] /[\mathrm{Rz}]
$$




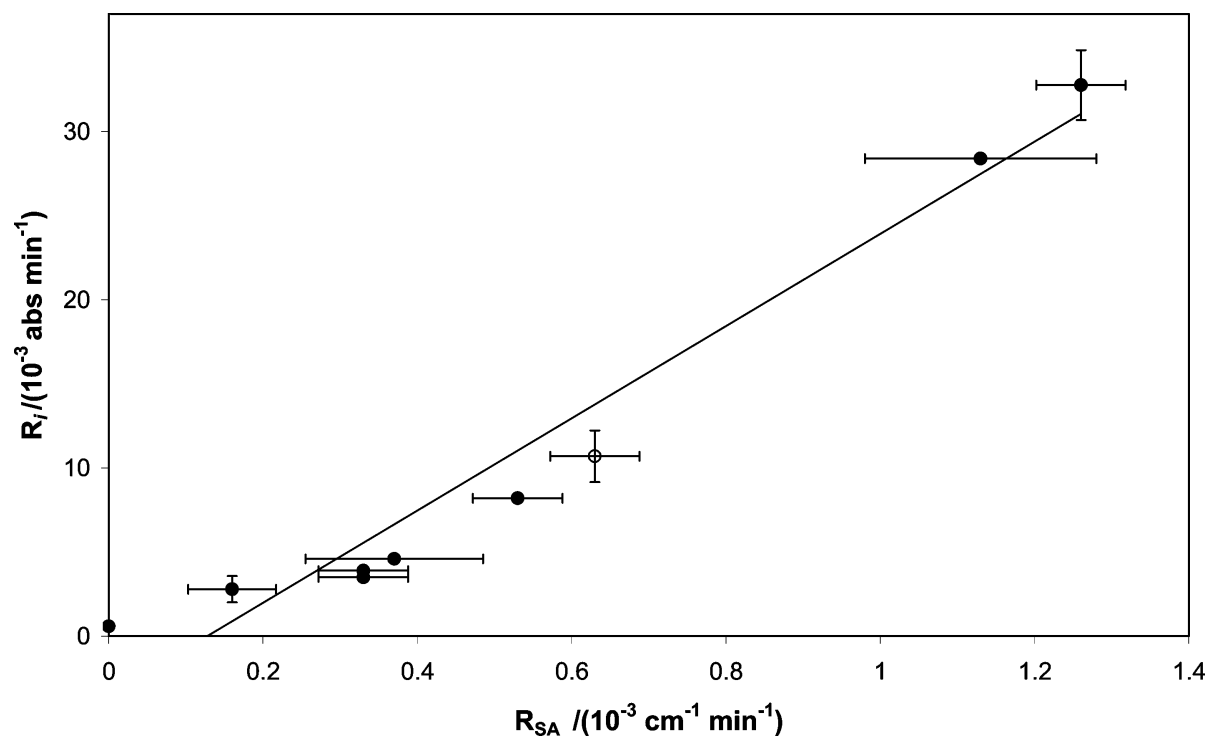

Figure 8. Plot of the $r_{\mathrm{i}}$ for a typical Rz indicator ink film and the initial rate of stearic acid removal, RSA, when deposited onto a series of CVD titania films exhibiting a range of photocatalytic activities. The open circle data point is that for Activ. In all cases the irradiance used was $0.32 \mathrm{~mW}$ $\mathrm{cm}^{-2}$.

Values of $f_{\mathrm{M}}$ and $f_{\mathrm{D}}$ can be readily calculated for any given film formulation, via the quadratic that arises from eq 4 , given knowledge of the values for $K\left(=0.05 \mathrm{mg}^{-1}\right)$ and the initial amount of Rz, i.e., [Rz], used in the formulation.

It might be expected that the dimer and the monomer of $\mathrm{Rz}$ contribute differently to the overall rate of $\mathrm{Rz}$ reduction, so that the overall initial rate, $r_{\mathrm{i}}$, is resolved into the two components, i.e.

$$
r_{\mathrm{i}}=f_{\mathrm{M} .} r_{\mathrm{iM}}+f_{\mathrm{D} .} r_{\mathrm{iD}}
$$

where $r_{\mathrm{iM}}$ and $r_{\mathrm{iD}}$ are the initial rates of dye reduction due to the monomer and dimer, respectively. Since values of $f_{\mathrm{M}}$ and $f_{\mathrm{D}}$ can be calculated for any film formulation using eqs 4 and 5 , it is possible to determine values for $r_{\mathrm{iM}}$ and $r_{\mathrm{iD}}$ of 27 and $-40 \times 10^{-3}$ abs units $\mathrm{min}^{-1}$, respectively, and these have been used to generate the good solid line fit to the $r_{\mathrm{i}}$ versus $[\mathrm{Rz}]$ data illustrated in Figure 6. The significant negative value for $r_{\mathrm{iD}}$ implies that the dimer of Rz not only does not contribute to the overall dye reduction of the monomer but also significantly impedes it, possibly by acting as a trap for the reducing species, - ROH, by mediating its oxidation by ambient oxygen.

The rate of Rz photoreduction by Activ films was also studied as a function of ambient temperature over the range $20-50{ }^{\circ} \mathrm{C}$ and found to increase slightly with increasing temperature. An Arrhenius plot of the kinetic data revealed a low activation energy of $9.1 \pm 0.1 \mathrm{~kJ} \mathrm{~mol}^{-1}$ for the dye reduction process, which is not atypical of many photocatalytic systems. ${ }^{1}$

The value of the Rz/glycerol/HEC ink as a photocatalyst indicator would be greatly enhanced if it can be shown that the initial rate of dye bleaching, $R_{\text {ink }}$, correlates well with the initial rate of stearic acid removal, $R_{\mathrm{SA}}$, which is so often used to assess the SPC activities of such films. Thus, a series of CVD-coated samples of anatase titania on glass with different SPC activities were prepared by varying the deposition conditions. These films, and a commercial sample of self-cleaning glass, Activ, were assessed for SPC activity using the stearic acid test. They were then cleaned and coated with the photocatalyst ink and assessed for SPC activity via the measured values of $r_{\mathrm{i}}$. A plot of the rate data arising from this work, i.e., $r_{\mathrm{i}}$, vs $R_{\mathrm{SA}}$, is illustrated in Figure 8 and reveals a good correlation between the two sets of rate data, the important difference being that for each sample

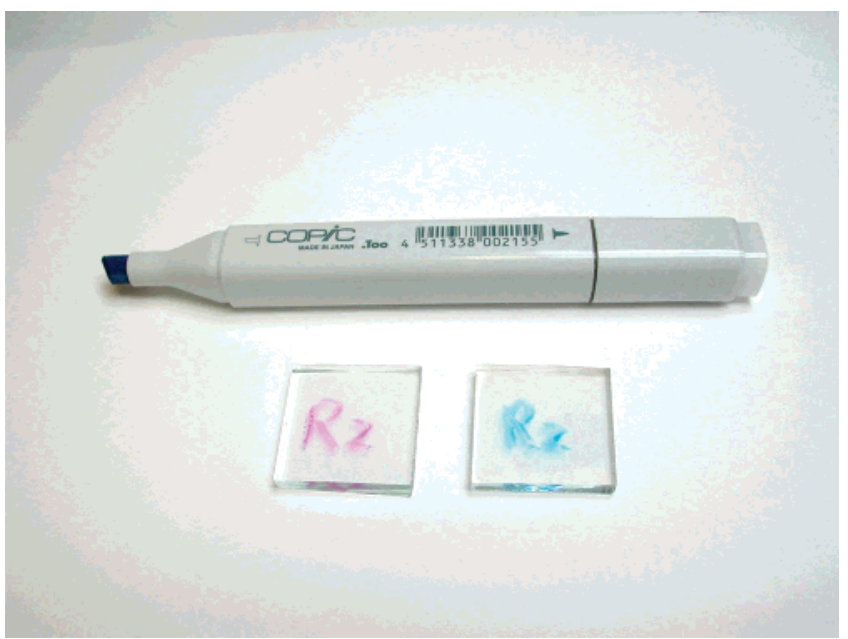

Figure 9. Photograph of a Rz photocatalyst indicator felt tip pen and pieces of Activ (left-hand side) and plain glass (right-hand side), inscribed "Rz" with the pen, after 3 min UV irradiation. The Activ glass turns the ca. $20 \mathrm{~nm}$ thick film of ink pink, whereas even prolonged irradiation of the ink on plain glass does not alter its originally blue color.

the $r_{\mathrm{i}}$ data were obtained in a few minutes, whereas the $R_{\mathrm{SA}}$ data required hours of illumination.

Finally, the efficacy of the photocatalyst indicator ink for work in the field was illustrated by placing $3 \mathrm{~mL}$ of the typical ink formulation in an empty felt-tipped pen and using it to write on a piece of plain and Activ glass. Upon exposure to 3 min of UVA light $\left(7.4 \mathrm{~mW} \mathrm{~cm}^{2}\right)$ the writing on the Activ-coated glass had changed from blue to pink. The writing on the plain glass remained blue even after prolonged $(6 \mathrm{~h})$ irradiation with the same UV source. Figure 9 shows a photograph of the pen and the two inscribed pieces of glass after irradiation. Given that most methods of assessing photocatalyst activity are slow and require expensive analytical equipment to service them, the $\mathrm{Rz}$ photocatalyst indicator ink reported here appears an attractive alternative, especially as it can be delivered using a simple felttipped pen or rubber stamp. Further work shows that even after a period of 6 months the $\mathrm{Rz}$ ink in the felt-tipped pen is as effective as new, indicating a significant stability and shelf life. 


\section{Conclusions}

The Rz photocatalyst ink provides a rapid and convenient method for assessing the SPC activity of a photocatalyst film. The ease with which such an assessment can be made using this intelligent ink makes it an ideal, inexpensive method to evaluate, in the field as well as the laboratory, the efficacy of photocatalytic materials including the many that have been utilized to create notable commercial products that are entering the market, such as self-cleaning glass, tiles, and paving stones. This method of assessment is of value, not only to those making photocatalytic materials, such as the researcher and the manufacturer, but also to the consumer who may wish to know if, after a few years, the product they purchased with its valueadded, self-cleaning coating is still effective.

Acknowledgment. We wish to thank Dr. Stephen Batchelor (Unilever Research, Port Sunlight, U.K.) for many helpful discussions regarding the possible mechanism of dye photoreduction.

\section{References and Notes}

(1) Mills, A.; LeHunte, S. J. Photochem. Photobiol., A: Chem. 1997, 108, 1. (b) Ollis, D. F.; Pelizetti, E.; Serpone, N. In Photocatalysis: Fundamentals and Applications; Serpone, N., Pelizetti, E., Eds.; WileyInterscience: New York, 1989. (c) Hoffmann, M. R.; Martin, S. T.; Choi, W.; Bahnemann, D. W. Chem. Rev. 1995, 95, 69. (d) Fox, M. A.; Dulay, M. T. Chem. Rev. 1993, 93, 34. (d) Kamat, P. V. Chem. Rev. 1993, 93 , 267.

(2) Mills, A. Lee, S. K. J. Photochem. Photobiol., A: Chem. 2002, 152,233 and references therein.

(3) Reisch, M. Chem. Eng. News 2001, 79, 8.

(4) Sanderson, K. D.; Mills, A.; Hurst, S.; Lepre, A.; McKittrick, T.; Rimmer D.; Ye, L. In SVC, $46^{\text {th }}$ Annual Technical Conference Proceedings; Society of Vacuum Coaters: Albuquerque, NM, 2003; p 203.

(5) Wang, R.; Hashimoto, K.; Fujishima, A.; Chikuni, M.; Kojima, E.; Kitamura, A.; Shimohigoshi, M.; Watanabe, T. Nature 1997, 388, 431.
(6) Fujishima, A.; Rao, T. N.; Tryk, D. A. J. Photochem. Photobiol., C: Photochem. Rev. 2001, 1,1 .

(7) Irie, H.; Hashimoto, K. In Environmental Photochemistry Part II; Boule, P., Bahnemann, D. W., Robertson, P. K. J., Eds.; Springer: Berlin, $2005 ;$ p 425.

(8) Sitkiewitz, S.; Heller, A. New J. Chem. 1996, 20, 233.

(9) Minabe, T.; Tryk, D. A.; Sawunyama, P.; Kikuchi, Y.; Hashimoto,

K.; Fujishima, A. J. Photochem. Photobiol., A: Chem. 2000, 137, 53.

(10) Sawunyama, P.; Jiang, L.; Fujishima, A.; Hashimoto, K. J. Phys. Chem. B 1997, 101, 11003

(11) Mills, A.; Lepre, A.; Elliott, N.; Bhopal, S.; Parkin, I. P.; O'Neill, S. A. J. Photochem. Photobiol., A: Chem. 2003, 160, 213.

(12) Remillard, J. T.; McBride, J. R.; Nietering, K. E.; Drews, A. R.; Zhang, X. J. Phys. Chem. B 2000, 104, 4440.

(13) Kemmitt, T.; Al-Salim, N. I.; Waterland, M.; Kennedy, V. J.; Markwitz, A. Curr. Appl. Phys. 2004, 4, 189.

(14) Tatsuma, T.; Tachibana, S.; Fujishima, A. J. Phys. Chem. B 2001, $105,6987$.

(15) Doushita, K.; Kawahara, T. J. Sol-Gel Sci. Technol. 2001, 22, 91.

(16) Mills, A.; Wang, J.; Lee, S. K. Chem. Commun. 2005, 2721.

(17) Tratnyek, P. G.; Reilkoff, T. E.; Lemon, A. W.; Scherer, M. M.; Balko, B. A.; Feik L. M.; Henegar, B. H. Chem. Educ. 2001, 6, 1.

(18) Green, F. J. The Sigma-Aldrich Handbook of Stains, Dyes and Indicators; Aldrich Chemical Co., Inc.: Milwaukee, WI, 1991; p 622.

(19) Bueno, C.; Villegas, M. L.; Bertolotti, S. G.; Previtali, C. M.; Neumann, M. G.; Encinas, M. V. Photochem. Photobiol. 2002, 76, 385

(20) Hykaway, N.; Sears, W. N.; Morisaki, H.; Morrison, S. R. J. Phys. Chem. 1986, 90, 663.

(21) Madelbaum, T. A.; Regazzoni, A. E.; Blesa, M. A.; Bilmes, S. A. J. Phys. Chem. B 1999, 103, 5505.

(22) Wang, C. Y.; Rabani, J.; Bahnemann, D. W.; Dohrmann, J. K. J. Photochem. Photobiol., A: Chem. 2002, 148, 169.

(23) Hilgendorff, M.; Bahnemann, D. W. In Environmental Aspects of Electrochemistry and Photoelectrochemistry; Tomkiewicz, M., Haynes, R., Yoneyama, H., Hori, Y., Eds.; The Electrochemical Society: Pennington, NJ, 1993; p 112.

(24) Mills, A.; Wang. J. J. Photochem. Photobiol., A: Chem. 1999, 127, 123.

(25) Sayilkan, F.; Asilturk, M.; Erdemoglu, S.; Akarsu, M.; Sayilkan, H.; Erdemoglu, M.; Arpac, V. Mater. Lett. 2006, 60, 230.

(26) Kwon, C. H.; Shin, H.; Kim, J. H.; Choi, W. S.; Yoon, K. H. Mater. Chem. Phys. 2004, 86, 78 .

(27) Van Keuren, E.; Schrof, W. Macromolecules 2003, 36, 5002. 